\title{
The Influence of Light Propagation Direction on the Stress-Induced Polarization Dependence of Silicon Waveguides
}

\author{
Min Huang
}

\begin{abstract}
This letter studies the effects of light propagation direction on the stress-induced polarization dependence of silicon-based waveguides. Silicon is an anisotropic material, so the stresses and the corresponding change of polarization dependence vary with the light propagation directions. The analyses show that when the light propagates in $\langle 010\rangle$ directions on (100) silicon, the stress-induced changes of refractive index and the birefringence in effective index are about $20 \%$ more sensitive to the stresses than those when the light propagates in $\langle 011\rangle$ directions.
\end{abstract}

Index Terms-Anisotropic media, birefringence, optical waveguides, polarization, silicon, stress.

\section{INTRODUCTION}

O ILICON-BASED optical waveguides have shown great application potential due to the major advantages of large refractive index contrast between the silicon core and the silica cladding, and the possibility to use the sophisticated microelectronic fabrication technology [1]. The major issue of the silicon waveguide is the large polarization dependence. One approach to tune the polarization dependence of the silicon ridge waveguide is to utilize the stresses caused by the upper cladding [2], [3]. Due to the photoelastic effect, the stress can change the refractive index, thus influencing the optical performance [4]. As silicon is considered as a cubic crystal, the material properties are anisotropic; therefore, the stress and the corresponding refractive index change depend on the light propagation direction in silicon waveguide. The light propagation direction continuously changes in the curved waveguides, so the response of the waveguide to the stress also changes constantly along the waveguide. In this letter, the effects of light propagation direction on the stress anisotropy in the waveguide core, and the corresponding changes of refractive index and the birefringence in effective index are studied.

\section{EFFECTS ON THE STRESS ANISOTROPY}

The structure studied in this letter is a ridge waveguide with a silicon core of $1 \times 1 \mu \mathrm{m}^{2}$ (etch depth $\times$ width) and a silica cladding layer with a thickness much larger than the waveguide core (Fig. 1). The stresses in the waveguide are induced by the thermal expansion mismatch between the silicon core and silica cladding. We take $x$-axis as the direction perpendicular to

Manuscript received February 6, 2006; revised April 3, 2006.

The author is with Silicon Technology Development, Texas Instruments Incorporated, Dallas, TX 75243 USA (e-mail: minhuang@ @i.com).

Digital Object Identifier 10.1109/LPT.2006.876746

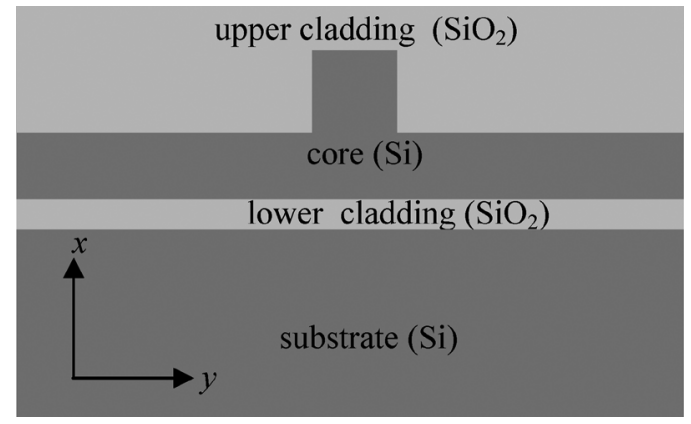

Fig. 1. Cross section of a Si ridge waveguide.

TABLE I

MATERIALS PROPERTIES OF (100) SiLICON

\begin{tabular}{cccccc}
\hline $\begin{array}{c}E_{11} \\
(\mathrm{GPa})\end{array}$ & $\begin{array}{c}E_{12} \\
(\mathrm{GPa})\end{array}$ & $\begin{array}{c}E_{44} \\
(\mathrm{GPa})\end{array}$ & $\begin{array}{c}C_{11} \\
\left(\mathrm{GPa}^{-1}\right)\end{array}$ & $\begin{array}{c}C_{12} \\
\left(\mathrm{GPa}^{-1}\right)\end{array}$ & $\begin{array}{c}C_{44} \\
\left(\mathrm{GPa}^{-1}\right)\end{array}$ \\
\hline 166 & 64 & 80 & -0.016 & 0.006 & -0.013 \\
\hline
\end{tabular}

The components of elasticity tensor $E$ are calculated from [5]; and the components of photoelastic tensor $C$ are calculated from [7].

the silicon wafer, $z$-axis as the light propagation direction, and $y$-axis as the waveguide width direction. The waveguide is fabricated on (100) plane, so $x$-axis is always [100] direction, but the light propagation direction, $z$-axis, can change from $\langle 010\rangle$ to $\langle 011\rangle$ directions. Silicon is considered as a cubic crystal. The elastic behavior is commonly described by three independent coefficients referred to the crystal axes. These coefficients need to be measured experimentally. For arbitrary axes, the elastic coefficients are determined from a tensor transformation. The details of the calculations of tensor transformation can be found in [5] and [6]. Under the coordinate system defined here, the stress-strain relation of silicon can be written as (1), shown at the bottom of the next page, where $\sigma$ and $\varepsilon$ are, respectively, stress tensor and strain tensor. $E$ is the elasticity tensor, which is determined by the light propagation direction (z-axis). $E_{11}$, $E_{12}$, and $E_{44}$, which are listed in Table I, are the components of the elasticity tensor when the light propagates in $\langle 010\rangle$ directions. $E_{11}^{\prime}$ and $E_{24}^{\prime}$ are determined by the light propagation direction, which can be calculated from tensor transformation [5], [6] using $E_{11}, E_{12}$, and $E_{44}$.

Due to the orientation dependent material properties, the stresses in the waveguide core depend on the light propagation direction. Fig. 2 shows the normalized stress anisotropy at the core center, $\left(\sigma_{x x}-\sigma_{y y}\right) /\left(\sigma_{x x}-\sigma_{y y}\right)_{\langle 010\rangle}$, as a function of 


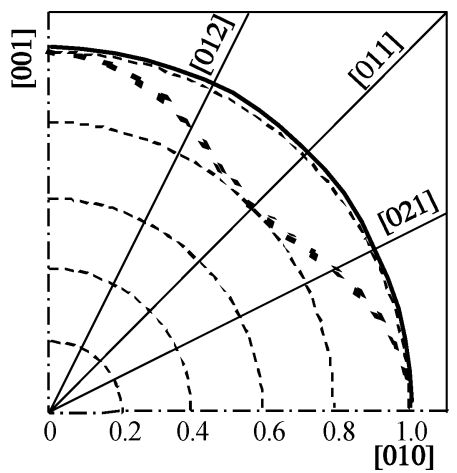

Fig. 2. Normalized stress anisotropy (solid line) and the corresponding normalized refractive index anisotropy (dashed line) as a function of the light propagation direction on (100) silicon.

light propagation direction. The curve is obtained by the finite element simulation, which is done with the ABAQUS commercial software (version 6.3, Hibbitt, Karlsson \& Sorensen, Pawtucket, RI, 2002). In the simulation, $E_{\text {silica }}=70 \mathrm{GPa}$, $\alpha_{\text {silica }}=0.5 \times 10^{-6} 1 / \mathrm{K}$, and $\alpha_{s i}=2.5 \times 10^{-6} 1 / \mathrm{K}$. We can see that when the light propagation direction changes, the change of stress anisotropy is small.

\section{EFFECTS ON THE STRESS-INDUCED REFRACTIVE INDEX ANISOTROPY}

With the change of the light propagation direction, the stress-induced refractive index is changed for the same wavegude structure and under the same stress. A similar method as the previous section is used to obtain the components of photoelastic relation as a function of light propagation direction. Under the same coordinate system as Section II, the photoelastic relation can be expressed as (2), shown at the bottom of the page, where $\Delta n$ is the tensor of the stress-induced refractive index change. $C$ is the photoelastic tensor, which is determined by the light propagation direction ( $z$-axis). $C_{11}$, $C_{12}$, and $C_{44}$, which are listed in Table I, are the components of the photoelastic tensor when the light propagates in $\langle 010\rangle$ directions [7]. Similar to the elasticity tensor, $C_{11}^{\prime}$ and $C_{24}^{\prime}$ are calculated from tensor transformation [5], [6] for different light propagation directions. As the dimension of the waveguide in the $z$ direction is much larger than those of other directions, we can treat it as a plane-strain problem, in which $\sigma_{y z}=0$. Thus, the refractive index anisotropy can be obtained from (2) as

$$
\begin{aligned}
\Delta n_{x x}-\Delta n_{y y}=\left(C_{12}-C_{11}\right) & \left(\sigma_{x x}-\sigma_{y y}\right) \\
& -\left(C_{11}-C_{11}^{\prime}\right)\left(\sigma_{y y}-\sigma_{z z}\right) .
\end{aligned}
$$

For isotropic materials, $C_{11}^{\prime}=C_{11}$; and for anisotropic materials, $C_{11}-C_{11}^{\prime}$ depends on the light propagation directions. When light propagates in $\langle 010\rangle$ directions, $C_{11}^{\prime}=C_{11}$. Based on the stresses obtained in Section II, the change of the normalized refractive index anisotropy at the center of the core, $\left(\Delta n_{x x}-\Delta n_{y y}\right) /\left(\Delta n_{x x}-\Delta n_{y y}\right)_{\langle 010\rangle}$, is calculated and shown in Fig. 2. We can see that the stress-induced refractive index change in the $\langle 010\rangle$ directions is about $20 \%$ larger than that in the $\langle 011\rangle$ directions. As the change of stress anisotropy is not sensitive to the light propagation direction, as shown in Section II, the change of the refractive index anisotropy is mainly caused by the multiplication of $\left(C_{11}-C_{11}^{\prime}\right)$ and $\left(\sigma_{y y}-\sigma_{z z}\right)$.

\section{EFFECTS ON THE STRESSED-INDUCED BIREFRINGENCE IN EFFECTIVE INDEX}

An analytical solution based on the effective index method and linear approximation to estimate the anisotropic stress-induced birefringence in effective index has been developed [8]. If the thickness of the upper silica claddings is much larger than the size of the waveguide core, the approach can be borrowed here and an approximate solution to estimate the stress-induced birefringence in effective index at different light propagation directions can be obtained as

$$
\begin{aligned}
\Delta n_{\mathrm{eff}}^{\prime} \approx & \frac{n_{h}^{2}}{n_{0} n_{\mathrm{eff}}} A_{w} \\
& \times\left\{\left(C_{12}-C_{11}\right)\left(\sigma_{x x}-\sigma_{y y}\right)\right. \\
& +\left[\left(C_{12}-C_{11}^{\prime}\right)-\frac{n_{0}^{2}}{n_{h}^{2}} A_{t}\left(C_{11}+C_{12}-2 C_{11}^{\prime}\right)\right] \\
& \left.\times\left(\sigma_{y y}-\sigma_{z z}\right)\right\}
\end{aligned}
$$

$$
\left(\begin{array}{l}
\sigma_{x x} \\
\sigma_{y y} \\
\sigma_{z z} \\
\sigma_{y z} \\
\sigma_{x z} \\
\sigma_{x y}
\end{array}\right)=\left(\begin{array}{cccccc}
E_{11} & E_{12} & E_{12} & 0 & 0 & 0 \\
E_{12} & E_{11}^{\prime} & E_{11}+E_{12}-E_{11}^{\prime} & E_{24}^{\prime} & 0 & 0 \\
E_{12} & E_{11}+E_{12}-E_{11}^{\prime} & E_{11}^{\prime} & -E_{24}^{\prime} & 0 & 0 \\
0 & E_{24}^{\prime} & -E_{24}^{\prime} & E_{11}+E_{44}-E_{11}^{\prime} & 0 & 0 \\
0 & 0 & 0 & 0 & E_{44} & 0 \\
0 & 0 & 0 & 0 & 0 & E_{44}
\end{array}\right)\left(\begin{array}{l}
\varepsilon_{x x} \\
\varepsilon_{y y} \\
\varepsilon_{z z} \\
\varepsilon_{y z} \\
\varepsilon_{x z} \\
\varepsilon_{x y}
\end{array}\right)
$$

$$
\left(\begin{array}{c}
\Delta n_{x x} \\
\Delta n_{y y} \\
\Delta n_{z z} \\
\Delta n_{y z} \\
\Delta n_{x z} \\
\Delta n_{x y}
\end{array}\right)=-\left(\begin{array}{cccccc}
C_{11} & C_{12} & C_{12} & 0 & 0 & 0 \\
C_{12} & C_{11}^{\prime} & C_{11}+C_{12}-C_{11}^{\prime} & C_{24}^{\prime} & 0 & 0 \\
C_{12} & C_{11}+C_{12}-C_{11}^{\prime} & C_{11}^{\prime} & -C_{24}^{\prime} & 0 & 0 \\
0 & C_{24}^{\prime} & -C_{24}^{\prime} & C_{11}+C_{44}-C_{11}^{\prime} & 0 & 0 \\
0 & 0 & 0 & 0 & C_{44} & 0 \\
0 & 0 & 0 & 0 & 0 & C_{44}
\end{array}\right)\left(\begin{array}{c}
\sigma_{x x} \\
\sigma_{y y} \\
\sigma_{z z} \\
\sigma_{y z} \\
\sigma_{x z} \\
\sigma_{x y}
\end{array}\right)
$$




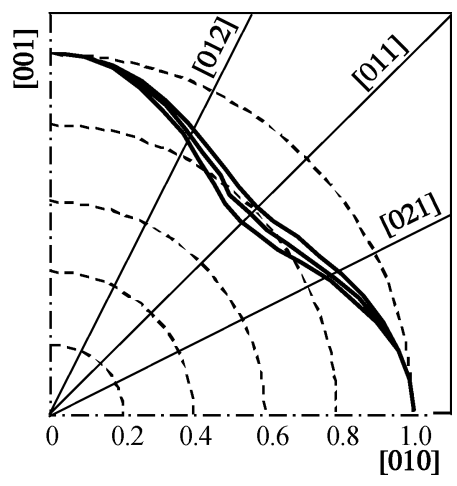

Fig. 3. Stress-induced normalized birefringence in effective index as a function of the light propagation direction on (100) silicon. The different lines are different core size (from the top to the bottom: $0.25,1.0$, and $0.5 \mu \mathrm{m}$ ).

where $\Delta n_{\text {eff }}^{\prime}$ is the stress-induced birefringence in effective index. $n_{\text {eff }}$ is the effective index under stress free state and $n_{h}$ is the TM effective index of the equivalent slab waveguide. $\lambda$ is the wavelength. $A_{w}$ and $A_{t}$ are coefficients defined in [8]. Fig. 3 shows the effect of light propagation direction on the stress-induced birefringence in effective index, $\Delta n_{\text {eff }}^{\prime} / \Delta n_{\mathrm{eff}_{\langle 010\rangle}^{\prime}}$. It can be seen that, similar to the change of refractive index, the stress-induced birefringence has a maximum in the $\langle 010\rangle$ directions and a minimum in the $\langle 011\rangle$ directions. The difference is about $20 \%$. This shows that the stress anisotropy can have some effects on the birefringence for different light propagation directions, while the quantitative number depends on the detailed waveguide structure, as the total waveguide birefringence in silicon-on-insulator is a complex function of geometric shape and the stress applied on the layer [2], [3].

\section{CONCLUDING REMARKS}

The effects of light propagation direction on the stress-induced polarization dependence in the silicon-based optical waveguides are studied. It is found that although stress anisotropy in the waveguide is not sensitive to the light propagation direction, the stress-induced refractive index anisotropy and the birefringence can have about $20 \%$ variations among different directions. If one wants to maximize the stress-induced polarization dependence of (100) silicon waveguide, one may design the waveguide with the light propagating in $\langle 010\rangle$ directions; if one wants to minimize the stress-induced polarization dependence of (100) silicon waveguide, one may design the waveguide with the light propagating in $\langle 011\rangle$ directions. In the curved waveguides, the stress-induced polarization dependence continuously varies with the waveguide direction. The anisotropy of the photoelastic properties should not be neglected when designing a sophisticated silicon-based waveguide. The work evokes the necessity to further study the anisotropy of the stress-induced polarization dependence of the real devices.

\section{REFERENCES}

[1] S. Janz, "Silicon-based waveguide technology for wavelength division multiplexing," Top. Appl. Phys., vol. 94, pp. 323-360, 2004.

[2] D.-X. Xu, P. Cheben, D. Dalacu, A. Delâ'ge, S. Janz, B. Lamontagne, M.-J. Picard, and W. N. Ye, "Eliminating the birefringence in silicon-oninsulator ridge waveguides by use of cladding stress," Opt. Lett., vol. 29, pp. 2384-2386, 2004.

[3] W. N. Ye, D.-X. Xu, S. Janz, P. Cheben, M.-J. Picard, B. Lamontagne, and N. G. Tarr, "Birefringence control using stress engineering in silicon-on-insulator (SOI) waveguides," J. Lightw. Technol., vol. 23, no. 3, pp. 1308-1318, Mar. 2005.

[4] M. Huang, "Stress effects on the performance of optical waveguides," Int. J. Solids Struct., vol. 40, pp. 1615-1632, 2003.

[5] J. J. Wortman and R. A. Evans, "Young's modulus, shear modulus, and Poisson's ratio in silicon and germanium," J. Appl. Phys., vol. 36, pp. 153-156, 1965.

[6] J. Turley and G. Sines, "The anisotropy of Young's modulus, shear modulus and Poisson's ratio in cubic materials," J. Phys. D, vol. 4, pp. 264-271, 1971.

[7] J. Xu and R. Stroud, Acousto-Optic Devices: Principles, Design, and Applications. New York: Wiley, 1992.

[8] M. Huang and X. Yan, "Analytical solutions to estimate the stress induced polarization shift and the temperature sensitivity of optical waveguides," J. Appl. Phys., vol. 95, pp. 2820-2826, 2004. 